\title{
Invasive multidrug-resistant non-typhoidal Salmonella infections in Africa: zoonotic or anthroponotic transmission?
}

\author{
Samuel Kariuki, ${ }^{1,2}$ Gunturu Revathi, ${ }^{3}$ Nyambura Kariuki, ${ }^{3}$ John Kiiru, ${ }^{1}$ \\ Joyce Mwituria, ${ }^{1}$ Jane Muyodi, ${ }^{1}$ Jane W. Githinji, ${ }^{4}$ Dorothy Kagendo, ${ }^{1}$ \\ Agnes Munyalo ${ }^{1}$ and C. Anthony Hart $^{2}$ \\ ${ }^{1}$ Centre for Microbiology Research, Kenya Medical Research Institute, PO Box 43640, Nairobi, \\ Kenya \\ ${ }^{2}$ Department of Medical Microbiology and Genito-Urinary Medicine, University of Liverpool, \\ Liverpool L69 3GA, UK \\ ${ }^{3}$ Department of Medical Microbiology, Kenyatta National Hospital, PO Box 20723, Nairobi, \\ Kenya \\ ${ }^{4}$ Central Veterinary Investigations Laboratory, PO Private Bag, Kabete, Kenya
}

Correspondence Samuel Kariuki skariuki@kemri.org

Received 15 October 2005 Accepted 26 January 2006

\begin{abstract}
In Africa, multidrug-resistant non-typhoidal salmonellae (NTS) are one of the leading causes of morbidity and high mortality in children under 5 years of age, second in importance only to pneumococcal disease. The authors studied NTS isolates from paediatric admissions at two hospitals in Nairobi, Kenya, and followed the index cases to their homes, where rectal swabs and stools from parents and siblings, and from animals in close contact, were obtained. The majority of NTS obtained from cases were Salmonella enterica serotype Typhimurium (106 out of 193; $54.9 \%)$ and Salmonella enterica serotype Enteritidis (64; $33.2 \%$ ), a significant proportion $(34 \cdot 2 \%)$ of which were multiply resistant to three or more antibiotics, including ampicillin, tetracycline, cotrimoxazole and chloramphenicol. Only $23.4 \%$ of NTS were fully susceptible to all 10 antibiotics tested. Of the 32 NTS obtained from contacts (nine adults and 23 children) at the homes of index cases, $21(65.6 \%)$ isolates were similar by antibiotic-susceptibility profiles and plasmid content, and their Xbal- and Spel-digested chromosomal DNA patterns were indistinguishable from those of the corresponding index cases. Only three out of $180(1 \cdot 7 \%)$ samples from environmental sources, including animals, soil, sewers and food, contained NTS matching those from corresponding index cases. The carriage of NTS in an asymptomatic population was represented by $6.9 \%$ of human contacts from 27 out of 127 homes sampled. This population of carriers may represent an important reservoir of NTS that would play a significant role in the epidemiology of community-acquired NTS bacteraemia in children.
\end{abstract}

\section{INTRODUCTION}

In industrialized countries, most individuals infected with non-typhoidal salmonellae (NTS) experience mild gastrointestinal illness involving diarrhoea, chills, abdominal cramps, fever, head and body aches, and nausea and vomiting. Infections are acquired as food poisoning and are usually self-limiting, and antimicrobial treatment is not recommended for uncomplicated illnesses (Fierer \& Swancutt, 2000; Gill \& Hamer, 2001). In most cases, outbreaks of NTS infection are caused by Salmonella enterica serotype Typhimurium (S. Typhimurium) and Salmonella enterica serotype Enteritidis (S. Enteritidis). A variety of

Abbreviation: NTS, non-typhoidal salmonellae. foods have been implicated as vehicles transmitting salmonellosis to humans, including poultry, beef, pork, eggs, milk, cheese, fish, shellfish, fresh fruit and juice, and vegetables (Espi et al., 2005; Mazurek et al., 2004; Varma et al., 2005). Contamination can occur at multiple steps along the food chain. Contact with farm animals, pets, reptiles and natural pet treats have also been associated with infection (Centers for Disease Control \& Prevention, 1999; Wall et al., 1996).

In all sub-Saharan African countries where they have been studied, NTS are the commonest or second-commonest cause of bacteraemia in children under 5 years of age (Bahwere et al., 2001; Berkley et al., 2005; Graham, 2002; Green \& Cheesbrough, 1993; Lepage et al., 1990). They are also the second-commonest cause of neonatal meningitis, 
the third-most-common cause of bacterial meningitis in children over 2 months of age in Malawi (Molyneux et al., 2003), and an important cause of septic arthritis (Lepage et al., 1990) and neonatal sepsis (Milledge et al., 2005). It is estimated that the minimum incidence of communityacquired NTS in rural and urban populations of children may be as high as 166 per 100000 per year for children under 5 years of age (Berkley et al., 2005; Mwangi et al., 2002). Of all admissions with febrile illness, NTS constitute $18 \%$ of cases and result in $28 \%$ mortality, compared to $5.7 \%$ mortality in children that do not have bacteraemia $(P<0 \cdot 001)$. In particular, multidrug-resistant $S$. Typhimurium causes serious outbreaks. For example, in Zaire (Cheesbrough et al., 1997; Green \& Cheesbrough, 1993) and Rwanda (Lepage et al., 1990), multidrug-resistant $S$. Typhimurium is the predominant cause of bacteraemic illness in children, while in Kenya this serotype is the predominant isolate in children with salmonellae bacteraemia (Kariuki et al., 2002, 2005; Mwangi et al., 2002). The source and mode of transmission of NTS in the African context have remained unknown, although it is thought that human-to-human transmission may play an important role, and we have been unable to demonstrate the presence in humans of multidrug-resistant NTS that are phenotypically and genotypically similar to the NTS found in food animals in Kenya (Kariuki et al., 2002). We report a prospective study that looked at NTS from children with bacteraemia and compared them with the serotypes and genotypes of NTS isolated from family contacts and environmental samples from the homes of index cases.

\section{METHODS}

Study patients. The study population comprised children from the suburbs of Nairobi, Kenya, aged less than 7 years admitted to two hospitals with suspected meningitis or sepsis during the period 2002-2004. Parents or caretakers of the children were asked to give consent for participation in the study and for follow-up to their homes for further specimen collection from contacts, animals and the environment. Research ethical approval was granted by the ethical review boards of the Kenya Medical Research Institute (KEMRI) and the Liverpool School of Tropical Medicine. All blood specimens were taken before antibiotic treatment commenced.

Specimens from contacts, animals and environment at home. Index cases from whom NTS were isolated were followed to their home areas, where more epidemiological data were obtained and the following specimens taken: rectal swabs or fecal specimens from siblings and parents of the index cases, water from nearby rivers and streams, and raw and cooked food from the homes of the index cases and from vendors in markets serving the areas. In addition, rectal swabs from farm animals, including pigs, chickens, cows and goats, were taken from around the homes of index cases or from neighbouring homes.

Laboratory procedures. Blood cultures and stool specimens were processed using standard techniques. Briefly, blood cultures were incubated in $5 \% \mathrm{CO}_{2}$ at $37^{\circ} \mathrm{C}$ for $18 \mathrm{~h}$, and if signs of bacterial growth were observed (air bubbles, turbidity, or both) they were subcultured on sheep blood agar and chocolate agar. The remaining blood cultures were reincubated for a further 7 days or until positive. Stools were processed by direct plating onto selective media
(XLD and brilliant green agar) (Oxoid) and by overnight enrichment in selective Selenite F broth (Oxoid) followed by plating onto $\mathrm{XLD}$ and brilliant green agar, and incubated in air at $37^{\circ} \mathrm{C}$ for $18 \mathrm{~h}$. NTS were identified using agglutinating antisera (Murex Biotech), and their identification was confirmed biochemically using API $20 \mathrm{E}$ strips (API System).

Environmental samples were initially cultured in RappaportVassiliadis soya broth (Oxoid) for enrichment. The broth culture was then subcultured onto XLD and brilliant green agar. NTS were identified as for blood and stool cultures. All NTS isolates were stored at $-70{ }^{\circ} \mathrm{C}$ on Protect beads (Technical Service Consultants) until analysed.

Antimicrobial susceptibility testing. Susceptibilities to various antimicrobials, ampicillin, coamoxiclav, tetracycline, cotrimoxazole, chloramphenicol, gentamicin, nalidixic acid, ciprofloxacin, cefuroxime and ceftriaxone, were determined by both controlled disc diffusion and measuring MICs using E-test strips (AB BIODISK) according to the manufacturer's instructions. Escherichia coli ATCC 25922 (with known MICs) was used as a control for potency of antibiotic discs and E-test strips. Disc diffusion susceptibility tests and MICs were interpreted according to the guidelines provided by the National Committee for Clinical Laboratory Standards (2002).

PFGE of macrorestricted chromosomal DNA. Chromosomal DNA from NTS isolates was prepared in agarose plugs as described previously (Kariuki et al., 2002). DNA in agarose plugs was digested using $25 \mathrm{U}$ each of $\mathrm{Xba \textrm {I }}$ or SpeI (Roche Diagnostics). PFGE of agarose plug inserts was then performed on a CHEF-DR III system (BioRad Laboratories) on a horizontal $1 \%$ agarose gel for $20 \mathrm{~h}$ at $120 \mathrm{~V}$, with a pulse time of $1-40 \mathrm{~s}$ at $14^{\circ} \mathrm{C}$. A lambda DNA digest consisting of a ladder ( $\sim 22$ fragments) of increasing size from 50 to $\sim 1000 \mathrm{~kb}$ was included as a DNA size standard. The gel was stained with ethidium bromide and photographed on a UV transilluminator (UVP Inc.). The restriction endonuclease digest patterns were compared, and their similarities were scored by the method of Tenover et al. (1995) and by using the Dice similarity coefficient formula $2 \mathrm{~h} /$ $(\mathrm{a}+\mathrm{b})$, where $\mathrm{h}$ is the number of matching bands, and $\mathrm{a}+\mathrm{b}$ is the total number of bands including matching and non-matching. Isolates that differed in their PFGE fragment patterns by one or two bands were regarded as closely related, as minor mutational changes would result in such patterns.

Mating experiments and plasmid extraction. Conjugation experiments were carried out in broth, as previously described by Kariuki et al. (2002), with E. coli $\mathrm{K}-12\left(\mathrm{Nal}^{\mathrm{R}}, \mathrm{Lac}^{+}\right)$as recipient. All NTS tested were susceptible to nalidixic acid. Transconjugants were selected on MacConkey agar (Oxoid) supplemented with nalidixic acid $\left(32 \mathrm{mg} \mathrm{l}^{-1}\right)$ and ampicillin or chloramphenicol $\left(32 \mathrm{mg} \mathrm{l}^{-1}\right.$ each). Plasmid DNA extraction was performed using a Plasmid Mini Prep kit (Qiagen) according to the manufacturer's instructions. Plasmids were separated by electrophoresis on horizontal $0.8 \%$ agarose gels at $100 \mathrm{~V}$ for $2 \mathrm{~h}$. Plasmid sizes were determined by coelectrophoresis with plasmids of known sizes from E. coli strains V517 (NCTC 50193) $(53 \cdot 7,7 \cdot 2,5 \cdot 6,3 \cdot 9,3 \cdot 0,2 \cdot 7,2 \cdot 1 \mathrm{~kb})$ and 39R861 (NCTC 50192) (147, 63, 43.5, 6.9 kb). DNA bands were visualized with a UV transilluminator (UVP) after staining with $0.05 \%$ ethidium bromide.

Statistical analysis. We compared proportions of characteristics in index cases against those in contacts and animal/environmental samples by using the chi square and Fisher's exact tests. 


\section{RESULTS AND DISCUSSION}

\section{Patients and bacterial isolates}

During the three-year study, a total of 193 NTS isolates were obtained from 2246 children with febrile illness who had blood taken for culture at admission (Table 1). The majority of cases $(68 \%)$ were in the age group less than 3 years old $(P<0 \cdot 0001$; odds ratio $(\mathrm{OR})=64 \cdot 834$; confidence interval $(\mathrm{CI})=3 \cdot 9-1078)$. A monthly mean of 22 admissions was recorded during the rainy months of May and June, which was significantly higher than a mean of 12 monthly admissions recorded for the rest of the year $(P<0 \cdot 0001$; $95 \% \mathrm{CI}=14 \cdot 21-24 \cdot 62)$. Although above the age of 3 years more boys than girls tended to present with severe NTS bacteraemia, this difference was not statistically significant $(P=0 \cdot 88 ; \mathrm{OR}=1 \cdot 04 ; 95 \% \mathrm{CI}=0 \cdot 587-1 \cdot 857)$. The main serotypes obtained from children with bacteraemia were $S$. Typhimurium (106;54.9\%) and S. Enteritidis $(64 ; 33 \cdot 2 \%)$, and there were $23(11.9 \%)$ isolates of other NTS serotypes (Table 2).

Prior to 1997, S. Typhimurium predominated (prevalence of $75 \%$ ) among cases of NTS bacteraemia in Kenya, and S.

Table 1. Distribution of NTS isolations among different age groups

\begin{tabular}{|lcc|}
\hline $\begin{array}{l}\text { Age group } \\
\text { (years) }\end{array}$ & $\begin{array}{c}\text { Number of NTS } \\
\text { isolates (\%) }\end{array}$ & $\begin{array}{c}\text { Male : female } \\
\text { ratio }\end{array}$ \\
\hline $0-1$ & $59(31 \%)$ & $0 \cdot 54: 0 \cdot 46$ \\
$1-3$ & $72(37 \%)$ & $0 \cdot 44: 0 \cdot 56$ \\
$3-5$ & $22(10 \%)$ & $0 \cdot 64: 0 \cdot 36$ \\
$5-7$ & $42(22 \%)$ & $0 \cdot 63: 0 \cdot 37$ \\
\hline
\end{tabular}

Enteritidis made up only $4.8 \%$ of cases. However, more recently (Kariuki et al., 2005), isolations of S. Enteritidis from blood cultures have steadily increased to a current prevalence of $40 \%$, probably due to changing lifestyles as more people rear chickens for eggs as a source of protein in the home. As in previous studies (Berkley et al., 2005), the majority $(68 \%)$ of the children admitted to hospital with severe bacteraemia were below 3 years of age, and nearly half of these were in the below 1 year age group. Similar observations were made with NTS isolations from contacts from homes of index cases. It is conceivable that the high rate of NTS bacteraemia in this age group is directly related to the low immune status of the children, especially immediately after weaning during the first year of life. In our study, the isolation of NTS from children with bacteraemia peaked during the rainy months of May and June, and this would coincide with reduced sanitary conditions in the homes and the environment in which the children live and play, particularly in the poor urban residential areas where the majority of the cases come from.

\section{Contacts and environmental isolates}

A total of 127 (66\%) parents and guardians gave written consent for follow-up of index cases to their homes for further specimen collection. Although a large proportion $(62 ; 48 \cdot 8 \%)$ of these homes were located within the three major slum areas around the city of Nairobi, most ( $84 \%)$ of the homes had access to treated running water. However, due to frequent water shortages in several city suburbs, most families stored water in cans, pots and plastic tanks for several days. A total of $66 \%$ of the population sampled did not have any animals at home. Among the 43 (34\%) families who had animals, the majority (76\%) kept five to ten chickens for eggs in the backyard, and one dog or cat. A few families $(28 ; 22 \%)$ grew vegetables in small gardens in their

Table 2. Numbers of NTS serotypes from cases, contacts and environmental samples

Values in parentheses show the number of cases as a percentage of the total.

\begin{tabular}{|c|c|c|c|c|}
\hline NTS serotype & \multicolumn{4}{|c|}{ Number of NTS isolates from: } \\
\hline S. Typhimurium & $106(54 \cdot 9)$ & $5(55 \cdot 5)$ & $12(52 \cdot 2)$ & $4(40)$ \\
\hline S. Enteritidis & $64(33 \cdot 2)$ & $4(44 \cdot 5)$ & $9(39 \cdot 1)$ & $2(20)$ \\
\hline Salmonella enterica serotype Newport & $4(0 \cdot 2)$ & - & - & - \\
\hline Salmonella enterica serotype Agona & $2(0 \cdot 1)$ & - & - & $1(10)$ \\
\hline Salmonella enterica serotype Choleraesuis & $2(0 \cdot 1)$ & - & - & $1(10)$ \\
\hline Salmonella enterica serotype Kiambu & $3(0 \cdot 2)$ & - & - & - \\
\hline Salmonella enterica serotype Derby & - & - & - & $1(10)$ \\
\hline
\end{tabular}

${ }^{\star}$ Environmental NTS included NTS from four soil samples, two cows, two sewer samples, one water sample and one vegetable sample. 
back yards for domestic use, but the majority bought supplies from vendors in neighbouring greengrocers and kiosks. Only 20 homes kept a milking cow for their own use and also sold any extra milk to neighbours.

From the 127 homes sampled we obtained a total of 32 $(6.9 \%)$ NTS from rectal swabs and faeces of 467 siblings and parents of index cases, and these came from 29 homes. Some $21(65 \cdot 6 \%)$ of these NTS serotypes were identical to serotypes obtained from the corresponding index cases. In addition, we obtained NTS from two cows from two homes and $8 / 180(4 \cdot 4 \%)$ NTS isolates from water, vegetables and soil samples from four other homes visited (Table 2). Four ( $2.2 \%$ prevalence) $S$. Typhimurium isolates from three soil samples and a water sample from three different homes also corresponded to the serotypes obtained from the index cases from the homes.

\section{Antibiotic susceptibility of NTS and resistance plasmids}

A total of $45(23 \cdot 4 \%)$ NTS from cases and $10(31 \cdot 3 \%)$ from contacts were fully susceptible to all antibiotics tested, while only $5 \%$ of NTS were resistant to only one antibiotic, mainly to ampicillin. A large proportion $(66 ; 34 \cdot 2 \%)$ of NTS from cases were resistant to three or more antibiotics; the most common resistance phenotypes were ampicillin, tetracycline and cotrimoxazole resistance in two-thirds of the isolates, and tetracycline, gentamicin and chloramphenicol or ampicillin resistance in $15 \%$ of the isolates. There were no significant differences in the prevalence of resistance between the two major serotypes $S$. Typhimurium and $S$. Enteritidis $(P=0 \cdot 087)$. Ciprofloxacin and ceftriaxone were the only antibiotics to which all NTS were fully susceptible. Table 3 shows the MIC values of NTS from cases for 10 commonly available antibiotics. The MIC values for the 21 NTS serotypes from 18 contacts, and from two soil samples and a water sample at the homes of index cases, were either identical $(89 \%)$ or within one dilution $(11 \%)$ of MICs of the NTS from corresponding cases.
Plasmid isolation and characterization was performed for all 148 antibiotic-resistant NTS (126 NTS from cases and 22 NTS from contacts at homes of index cases). All ampicillinresistant and multidrug-resistant NTS contained a 100$110 \mathrm{~kb}$ plasmid in addition to two to four plasmids between 5 and $12 \mathrm{~kb}$. The large $100-110 \mathrm{~kb}$ plasmid was selftransferable to E. coli K-12, carrying with it resistance to ampicillin and combinations of resistances to tetracycline, chloramphenicol and cotrimoxazole.

In Africa and most other developing regions, multidrug resistance, particularly to commonly available antibiotics, remains a major challenge for the healthcare system (Bonfiglio et al., 2002; Kariuki et al., 2005; World Health Organization, 2000). In particular, multidrug-resistant NTS have caused life-threatening invasive disease outbreaks in children in many African countries, including Zaire (Cheesbrough et al., 1997; Green \& Cheesbrough, 1993), Rwanda (Lepage et al., 1990), Nigeria (Adejuyigbe et al., 2004) and Malawi (Graham, 2002; Milledge et al., 2005). In Tanzania (Vaagland et al., 2004), multidrug-resistant $S$. Enteritidis was the main isolate in an outbreak of nosocomial meningitis in children from a rural community, while in the Central African Republic (Kassa-Kelembho et al., 2003), multidrug-resistant $S$. Typhimurium was the predominant cause of community-acquired bacteraemic illness in both children and in adults. In our study, only $23.4 \%$ of NTS from cases of severe bacteraemia in children were fully susceptible to all antibiotics tested, while a large proportion $(66 ; 34 \cdot 2 \%)$ of the NTS were multiply resistant to three or more commonly available antibiotics, including ampicillin, chloramphenicol, cotrimoxazole and tetracycline. It is noteworthy that the availability of these antibiotics over the counter and without prescription mainly for self-treatment of suspected infection in humans may have played a major role in the high prevalence of the multidrugresistance phenotype. In addition, the availability of cheaper generic drugs of variable quality for treatment of bacterial infections may also have contributed to the increased levels

Table 3. MIC results using the E-Test of 10 antimicrobial agents for 193 NTS isolates from paediatric wards at two hospitals in Nairobi, Kenya (2002-2004)

\begin{tabular}{|c|c|c|c|c|c|}
\hline \multirow[t]{2}{*}{ Antimicrobial agent } & \multicolumn{5}{|c|}{$\operatorname{MIC}\left(\mu \mathrm{g} \mathrm{ml}^{-1}\right)$} \\
\hline & Range & Mode & $\mathrm{MIC}_{50}$ & $\mathrm{MIC}_{90}$ & Percentage resistant \\
\hline Coamoxiclav & $0 \cdot 75-256$ & 4 & 1 & 16 & 8 \\
\hline Cefuroxime & $2-256$ & $>256$ & 8 & 32 & 30 \\
\hline Ceftriaxone & $0 \cdot 094-16$ & $0 \cdot 064$ & $0 \cdot 5$ & 2 & 0 \\
\hline Chloramphenicol & $0 \cdot 19-256$ & $>256$ & 4 & 32 & 26 \\
\hline Tetracycline & $0 \cdot 064-256$ & 3 & 16 & 128 & 51 \\
\hline Nalidixic acid & $1 \cdot 5-256$ & 3 & 3 & 3 & 12 \\
\hline Ciprofloxacin & $0 \cdot 064-4$ & $0 \cdot 16$ & $0 \cdot 06$ & $0 \cdot 125$ & 0 \\
\hline
\end{tabular}


of resistance. Multidrug-resistant NTS strains causing severe bacteraemia in children also pose a major public health concern in Kenya, since they are more difficult to treat, as more expensive and less readily available drugs such as ciprofloxacin and ceftriaxone will be required.

\section{Genotypes of NTS from bacteraemia and from contacts from homes of index cases}

Genotyping by PFGE was performed for the two common serotypes $S$. Typhimurium (106 isolates) and $S$. Enteritidis (64 isolates) from the cases, and a corresponding $21 \mathrm{~S}$. Typhimurium and $15 \mathrm{~S}$. Enteritidis isolates from homes of index cases. Fragments less than $100 \mathrm{~kb}$ were excluded from analysis, as they may have been plasmids, especially from the antibiotic-resistant NTS. XbaI- and SpeI-digested chromosomal DNA fragment analysis revealed a total of three main patterns (comprising $88 \%$ of all isolates; $65 \%$ in pattern 1 , $11 \%$ in pattern 2 and $6 \%$ in pattern 3 ) for $S$. Typhimurium and two main patterns for $S$. Enteritidis (comprising $84 \%$ of all isolates; $72 \%$ in pattern 1 and $12 \%$ in pattern 2) (Fig. 1), with digest fragments ranging in size from 50 to $650 \mathrm{~kb}$ and within a one to two band difference for each PFGE pattern. The remaining strains within each of the two main NTS serotypes were distantly related to the main clones (between three and five band differences between 50 and $200 \mathrm{~kb}$ in size). A total of 28/42 NTS (including four environmental isolates from soil and water) from homes of index cases produced digest patterns that were indistinguishable from those of NTS from corresponding index cases (Fig. 2). Another four $S$. Typhimurium isolates from healthy children at home were closely related (less than three band differences) to corresponding NTS from cases.

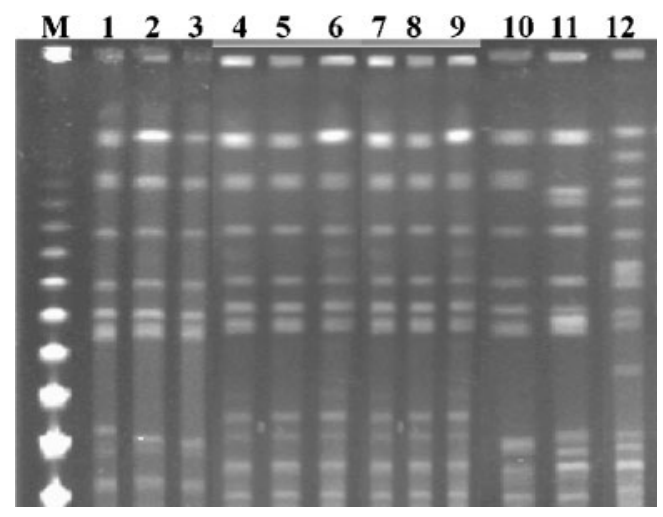

Fig. 1. Representative PFGE patterns of Xbal-digested $S$. Typhimurium from cases of children with bacteraemia. Lane $M$, $50 \mathrm{~kb}$ molecular size standard; lanes 1-9, S. Typhimurium from main group 1 PFGE pattern; lanes 10 and 11, S. Typhimurium from group 2 PFGE pattern; lane 12, $S$. Typhimurium from group 3 PFGE pattern. Using either of the two enzymes Xbal and Spel, PFGE patterns of the isolates were within one to three band differences of the three main groups.
From our study, it is apparent that a limited number of clones of NTS have been stably maintained in the community over the last four years. From the study population followed in 127 homes of index cases, an NTS carriage rate in asymptomatic individuals of $6.9 \%$ was obtained. This represents a significant proportion of the community in the study area. From this population of carriers, $65.6 \%$ of NTS isolates were clonally related to those found causing bacteraemia in children admitted to hospital in Nairobi.

\section{Epidemiology of NTS}

Most studies, particularly in industrialized countries, have implicated farm animals as the main reservoirs of NTS, and infections have often been associated with food contamination, either in homes or in the food production industry (Angulo et al., 2000; Hsueh et al., 2004; Tauxe, 1997). However, the sources and transmission dynamics of NTS in Africa have remained unknown, and it was only speculated that person-to-person transmission, in addition to sporadic foodborne outbreaks, played a significant role in community-acquired NTS bacteraemia in children. Our study observed that a significant number (a prevalence of $6.9 \%$ ) of NTS from siblings and parents of index cases were of the same serotype and antibiotic-susceptibility profiles, and were clonal in origin. In this portion of the community, NTS behaved in a similar manner to typhoid in terms of the characteristics of asymptomatic carriage. It is probable that the finding of NTS in family contacts of an index case represents either a situation in which all family members acquired the bacterium at the same time or that the index case became infected from other family members or vice versa. NTS may also precipitate serious infection in these

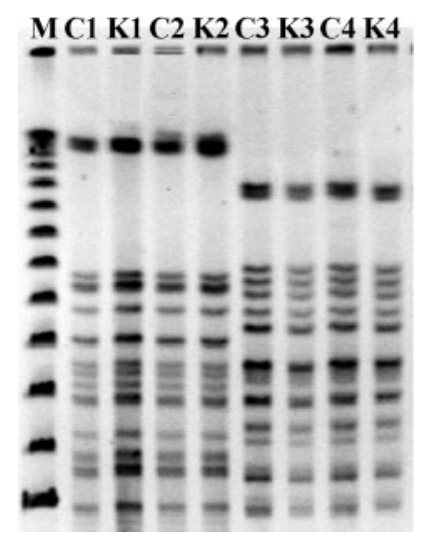

Fig. 2. Representative PFGE patterns of Spel digests of NTS from cases and from corresponding family contacts at homes of index cases. Lane M, $50 \mathrm{~kb}$ molecular size standard; lanes $\mathrm{C} 1$ and $\mathrm{C} 2, \mathrm{~S}$. Enteritidis, and lanes $\mathrm{C} 3$ and $\mathrm{C} 4, \mathrm{~S}$. Typhimurium from cases from Hospital A obtained over an eight-month period; lanes $\mathrm{K} 1$ and $\mathrm{K} 2, \mathrm{~S}$. Enteritidis, and lanes K3 and K4, S. Typhimurium from children from homes of corresponding index cases followed up within 2-3 weeks of the discharge of the case from hospital. 
carriers if malnutrition, underlying illness and immunosuppression are present, and the carriers may also shed NTS into the environment, providing a possible source of infection to other children in the home. It is possible that transmission may occur through food, water or contamination of surfaces within the home. Significantly, this study did not find a major reservoir of NTS in animals or food in the homes of index cases; the only NTS that matched those from corresponding index cases were all isolated from soil samples and a water sample.

In studies of sepsis in children aged between 6 months and 2 years from Malawi (Graham, 2002; Milledge et al., 2005), it has been observed that increased numbers of cases of NTS bacteraemia during the rainy season are strongly associated with an upsurge in cases of malaria and anaemia. However, our study was based in a highland area where malaria is not endemic, and hence this association may not apply. In another study, Cherubin et al. (1969) have found that an association between salmonellosis and low-income areas of New York City was particularly marked for childhood cases of $S$. Typhimurium. The authors hypothesize that $S$. Typhimurium maintains itself by human-to-human transmission, whereas other serotypes are introduced into the community by food vehicles. However, in our study, both $S$. Typhimurium and $S$. Enteritidis were isolated from contacts in proportions almost equivalent to those among cases, lending credence to the supposition that human-to-human transmission is important for both serotypes. In subSaharan Africa, it will be important to monitor carriage of multidrug-resistant NTS in the community as a possible source of severe bacteraemic infections in children.

\section{ACKNOWLEDGEMENTS}

We thank the Director, Kenya Medical Research Institute, for permission to publish this work. We are grateful to Rhoda Njagi for data management, and James Waithaka and Charles Lang'at for assistance in follow-up field studies. S. K. is supported by a Wellcome Trust Training Fellowship.

\section{REFERENCES}

Adejuyigbe, E. A., Ako-Nai, A. K. \& Adisa, B. (2004). Bacterial isolates in the sick young infant in Ile-Ife, Nigeria. J Trop Pediatr 50, 323-327.

Angulo, F. J., Johnson, K. R., Tauxe, R. V. \& Cohen, M. L. (2000). Origins and consequences of antimicrobial-resistant nontyphoidal Salmonella: implications for the use of fluoroquinolones in food animals. Microb Drug Resist 6, 77-83.

Bahwere, P., Levy, J., Hennart, P., Donnen, P., Lomoyo, W., Dramaix-Wilmet, M., Butzler, J. P. \& De Mol, P. (2001). Community-acquired bacteremia among hospitalized children in rural central Africa. Int J Infect Dis 5, 180-188.

Berkley, J. A., Lowe, B. S., Mwangi, I. \& 11 other authors (2005). Bacteremia among children admitted to a rural hospital in Kenya. $N$ Engl J Med 352, 39-47.
Bonfiglio, G., Simpore, J., Pignatelli, S., Musumeci, S. \& Solinas, M. L. (2002). Epidemiology of bacterial resistance in gastro-intestinal pathogens in a tropical area. Int J Antimicrob Agents 20, 387-389.

Centers for Disease Control and Prevention (1999). Reptileassociated salmonellosis-selected states, 1996-1998. Morbid Mortal Wkly Rep 48, 1009-1013.

Cheesbrough, J. S., Taxman, B. C., Green, S. D., Mewa, F. \& Numbi, I. (1997). A clinical definition for invasive Salmonella infection in African children. Pediatr Infect Dis J 16, 277-283.

Cherubin, C. E., Fodor, T., Denmark, L., Master, C., Fuerst, H. T. \& Winter, J. (1969). The epidemiology of salmonellosis in New York City. Am J Epidemiol 90, 112-125.

Espi, E., De Valk, H., Vaillant, V., Quelquejeu, N., Le Querrec, F. \& Wei, F. X. (2005). An outbreak of multidrug-resistant Salmonella enterica serotype Newport infections linked to the consumption of imported horse meat in France. Epidemiol Infect 133, 373-376.

Fierer, J. \& Swancutt, M. (2000). Non-typhoid Salmonella: a review. Curr Clin Top Infect Dis 20, 134-157.

Gill, C. J. \& Hamer, D. H. (2001). Foodborne illnesses. Curr Treat Options Gastroenterol 4, 23-38.

Graham, S. M. (2002). Salmonellosis in children in developing and developed countries and populations. Curr Opin Infect Dis 15, 507-512.

Green, S. D. \& Cheesbrough, J. S. (1993). Salmonella bacteraemia among young children at a rural hospital in western Zaire. Ann Trop Paediatr 13, 45-53.

Hsueh, P. R., Teng, L. J., Tseng, S. P. \& 20 other authors (2004). Ciprofloxacin-resistant Salmonella enterica Typhimurium and Choleraesuis from pigs to humans, Taiwan. Emerg Infect Dis 10, 60-68.

Kariuki, S., Revathi, G., Gakuya, F., Yamo, V., Muyodi, J. \& Hart, C. A. (2002). Lack of clonal relationship between non-typhi Salmonella strain types from humans and those isolated from animals living in close contact. FEMS Immunol Med Microbiol 33, 165-171.

Kariuki, S., Revathi, G., Muyodi, J., Mwituria, J., Munyalo, A., Kagendo, D., Murungi, L. \& Hart, C. A. (2005). Increasing prevalence of multidrug-resistant non-typhoidal salmonellae, Kenya, 1994-2003. Int J Antimicrob Agents 25, 39-45.

Kassa-Kelembho, E., Mbolidi, C. D., Service, Y. B., Morvan, J. \& Minssart, P. (2003). Bacteremia in adults admitted to the Department of Medicine of Bangui Community Hospital (Central African Republic). Acta Trop 89, 67-72.

Lepage, P., Bogaerts, J., Van Goethem, C., Hitimana, D. G. \& Nsengumuremyi, F. (1990). Multiresistant Salmonella typhimurium systemic infection in Rwanda. Clinical features and treatment with cefotaxime. J Antimicrob Chemother 26, 53-57.

Mazurek, J., Salehi, E., Propes, D., Holt, J., Bannerman, T., Nicholson, L. M., Bundesen, M., Duffy, R. \& Moolenaar, R. L. (2004). A multistate outbreak of Salmonella enterica serotype Typhimurium infection linked to raw milk consumption - Ohio, 2003. J Food Prot 67, 2165-2170.

Milledge, J., Calis, J. C., Graham, S. M. \& 8 other authors (2005). Aetiology of neonatal sepsis in Blantyre, Malawi: 1996-2001. Ann Trop Paediatr 25, 101-110.

Molyneux, E. M., Tembo, M., Kayira, K. \& 7 other authors (2003). The effect of HIV infection on paediatric bacterial meningitis in Blantyre, Malawi. Arch Dis Child 88, 1112-1118.

Mwangi, I., Berkley, J., Lowe, B., Peshu, N., Marsh, K. \& Newton, C. R. (2002). Acute bacterial meningitis in children admitted to a rural Kenyan hospital: increasing antibiotic resistance and outcome. Pediatr Infect Dis J 21, 1042-1048.

National Committee for Clinical Laboratory Standards (2002). Performance standards for antimicrobial susceptibility testing; twelfth 
informational supplement. Approved standard M100-S12. Wayne, PA: National Committee for Clinical Laboratory Standards.

Tauxe, R. V. (1997). Emerging food-borne diseases: an evolving public health challenge. Emerg Infect Dis 3, 425-434.

Tenover, F. C., Arbeit, R. D., Goering, R. V., Mickelsen, P. A., Murray, B. E., Persing, D. H. \& Swaminathan, B. (1995). Interpreting chromosomal DNA restriction patterns produced by pulsed-field gel electrophoresis: criteria for bacterial strain typing. J Clin Microbiol 33, 2233-2239.

Vaagland, H., Blomberg, B., Kruger, C., Naman, N., Jureen, R. \& Langeland, N. (2004). Nosocomial outbreak of neonatal Salmonella enterica serotype Enteritidis meningitis in a rural hospital in northern Tanzania. BMC Infect Dis 4, 35-37.

Varma, J. K., Greene, K. D., Ovitt, J., Barrett, T. J., Medalla, F. \& Angulo, F. J. (2005). Hospitalization and antimicrobial resistance in Salmonella outbreaks, 1984-2002. Emerg Infect Dis 11, 943-946.

Wall, P. G., Threlfall, E. J., Ward, L. R. \& Rowe, B. (1996). Multiresistant Salmonella typhimurium DT104 in cats: a public health risk. Lancet 348, 471.

World Health Organization (2000). Overcoming antimicrobial resistance. Publication code WHO/CDS/2000.2. Geneva: World Health Organization. 